\title{
A Critically Appraised Topic on the Tuck Jump Assessment: Does the Tuck Jump Assessment Demonstrate Interrater and Intrarater Reliability in Healthy Individuals?
}

\author{
Marissa L. Mason, Marissa N. Clemons, Kaylyn B. LaBarre, Nicole R. Szymczak, and Nicole J. Chimera
}

\begin{abstract}
Clinical Scenario: Lower-extremity injuries in the United States costs millions of dollars each year. Athletes should be screened for neuromuscular deficits and trained to correct them. The tuck jump assessment (TJA) is a plyometric tool that can be used with athletes. Clinical Question: Does the TJA demonstrate both interrater and intrarater reliability in healthy individuals? Summary of Key Findings: Four of the 5 articles included in this critically appraised topic showed good to excellent reliability; however, caution should be taken in interpreting these results. Although composite scores of the TJA were found to be reliable, individual flaws do not demonstrate reliability on their own, with the exception of knee valgus at landing. Aspects of the TJA itself, including rater training, scoring system, playback speed, volume, and number of views allotted, need to be standardized before the reliability of this clinical assessment can be further researched. Clinical Bottom Line: The TJA has shown varying levels of reliability, from poor to excellent, for both interrater and intrarater reliability, given current research. Strength of Recommendation: According to the Centre for Evidence Based Medicine levels of evidence, there is level 2b evidence for research into the reliability of the TJA. This evidence has been demonstrated in elite, adolescent, and college-level athletics in the United Kingdom, Spain, and the United States. The recommendation of level $2 \mathrm{~b}$ was chosen because these studies utilized cohort design for interrater and intrarater reliability across populations. An overall grade of B was recommended because there were consistent level 2 studies.
\end{abstract}

Keywords: rehabilitation, sport management, sports physiotherapist, tuck jump assessment, testing and measurement

\section{Clinical Scenario}

Lower-extremity (LE) injuries are common in the athletic population, especially injuries to the anterior cruciate ligament (ACL) ${ }^{1}$ These injuries can be career ending for athletes and result in costs in the United States of approximately 1 billion dollars annually. ${ }^{2}$ The large number of LE injuries and ACL injuries in particular may be able to be reduced with proper screening, which could reduce costs to the US health care system. ${ }^{2}$ Currently, plyometric screening tests include the landing error scoring system, ${ }^{3}$ the drop jump video screening test, ${ }^{4}$ and the tuck jump assessment (TJA). ${ }^{5-7}$ The TJA is a clinician-friendly tool that requires minimal equipment, is time efficient (it only takes $10 \mathrm{~s}$ to administer), and evaluates jumping performance for 10 flaws. Two-dimensional video (frontal and sagittal) is taken of an individual performing repeated tuck jumps for 10 seconds, and the rater watches the videos to retrospectively score the TJA for the presence or absence of each flaw. If a flaw is present one or more times, a score of 1 is given, and if a flaw is absent during the 10 seconds, a score of 0 is given. An athlete may earn a score between 0 and 10; a score of 10 represents the poorest neuromuscular performance, with all flaws present, and a score of 0 represents the best performance, with no flaws present. ${ }^{5-7}$

\section{Focused Clinical Question}

Does the TJA demonstrate both interrater and intrarater reliability in healthy individuals?

Mason, Clemons, LaBarre, and Szymczak were physical therapy students at Daemen College, Amherst, NY at the time of the research. Chimera is with the Department of Kinesiology, Brock University, St. Catharines, ON, Canada. Chimera (nchimera@ brocku.ca) is corresponding author.

\section{Summary of Search, Best Evidence Appraised, and Key Findings:}

- Five cohort studies ${ }^{8-12}$ were found in the literature review that met the inclusion criteria for this critically appraised topic.

- Three studies ${ }^{8-10}$ involved participants who were healthy, collegeaged athletes, and 2 studies $^{11,12}$ involved healthy youth athletes.

- Two of the studies ${ }^{9,10}$ provided a full description of the raters. Dudley et al $^{9}$ included 5 raters: a physical therapist with a Doctor of Physical Therapy and 4 years of clinical experience, a certified strength and conditioning coach with 7 years of clinical experience, a certified athletic trainer with 17 years of clinical experience, a third-year Doctor of Physical Therapy student, and a first-year Doctor of Physical Therapy student. Mayhew et $\mathrm{al}^{10}$ included 4 raters: 2 physical therapists with 10 and 11 years of experience, respectively, and 2 strength and conditioning coaches with 1 and 11 years of experience, respectively. Another study stated that the raters were certified strength and conditioning coaches with more than 5 years of experience each. ${ }^{12}$ In addition, 2 of the studies did not mention any education of the raters or related clinical experience. ${ }^{8,11}$

- Interrater reliability was determined in 4 of the studies, ${ }^{8-10,12}$ and intrarater reliability was also determined in 4 studies. ${ }^{8,9,11,12}$

\section{Clinical Bottom Line}

There is insufficient evidence to determine the reliability of the TJA in healthy individuals. According to Centre for Evidence Based Medicine levels of evidence, there is level $2 \mathrm{~b}$ evidence for research 
into the reliability of the TJA. This evidence has been demonstrated in elite, adolescent, and college-level athletics in the United Kingdom, Spain, and the United States.

\section{Search Strategy}

\section{Terms Used to Guide Search Strategy}

- Patient/Client group: healthy subjects OR athletes OR collegiate athletes

- Intervention: tuck jump assessment OR TJA

- Comparison: no comparison

- Outcomes: reliability OR interrater reliability OR intrarater reliability OR like term

\section{Sources of Evidence Searched}

- PubMed

- MEDLINE

- CINAHL

- SPORTDiscus

\section{Inclusion and Exclusion Criteria}

\section{Inclusion Criteria}

- Studies with healthy participants

- English language studies published from May 2012 to October 2018

- Oxford Centre for Evidence-Based Medicine rating of $2 b$ or higher

- Articles that have been peer reviewed

\section{Exclusion Criteria}

- Studies that did not evaluate interrater and/or intrarater reliability

\section{Results of Search}

Five relevant studies were found and are presented in Table 1. Interrater reliability was determined in 4 of the studies, ${ }^{8-10,12}$ and intrarater reliability was also determined in 4 of the studies. ${ }^{8,9,11,12}$

\section{Best Evidence}

The 5 studies chosen for this critically appraised topic were identified as the best match when considering the inclusion and exclusion criteria. These 5 studies investigated interrater and intrarater reliability (Table 2).

\section{Table 1 Summary of Study Designs of Articles}

\begin{tabular}{|c|c|c|}
\hline $\begin{array}{l}\text { Level of } \\
\text { evidence }\end{array}$ & Study design & Reference \\
\hline $2 b$ & Cohort study & Herrington et $\mathrm{al}^{8}$ \\
\hline $2 b$ & Cohort study & Dudley et $\mathrm{al}^{9}$ \\
\hline $2 b$ & Cohort study & Mayhew et al ${ }^{10}$ \\
\hline $2 b$ & Cohort study & Read et $\mathrm{al}^{11}$ \\
\hline $2 b$ & Cohort study & Fort-Vanmeerhaeghe et al ${ }^{12}$ \\
\hline
\end{tabular}

\section{Implications for Practice, Education, and Future Research}

Musculoskeletal conditions were ranked as the third-highest expenditure in health care spending in 2013, accounting for 190 billion health care dollars spent that year. ${ }^{13}$ A large portion of musculoskeletal care involves treatment of LE injuries. Of all sports-related injuries, $66 \%$ are LE injury injuries, with knee injuries being most common. ${ }^{14}$ A study conducted by Herzog et $\mathrm{al}^{15}$ found that between 2005 and 2013 the immediate cost for 229,446 ACL surgeries was over 2 billion dollars. This statistic does not account for the rehabilitation process following the surgery. ACL injuries can be career-ending injuries for many athletes. Therefore, athletes' risk for LE injury should be recognized, treated, and prevented via clinical assessment tools and strength and endurance training programs. This will not only prevent devastating consequences to athletic careers but also assist in reducing health care costs in our nation.

To reduce the risk of career-ending ACL injuries, several plyometric screening tools been developed, including the landing error scoring system, ${ }^{3}$ the drop jump video screening test,${ }^{4}$ and the TJA. ${ }^{5-7}$ Tools like the landing error scoring system and the drop jump video screening are more time consuming than the TJA, which only takes 10 seconds to conduct. The TJA has been deemed clinician friendly by researchers of the tool because it requires minimal equipment. ${ }^{9,15}$

The TJA starts with camera set up in both the frontal and sagittal planes. ${ }^{7}$ Two pieces of tape on the ground, $35 \mathrm{~cm}$ apart, indicate the starting and finishing point for the athlete's feet. Myers et $\mathrm{al}^{7}$ described that the athlete should jump straight up and at the highest point of the jump bring the knees as high as possible. The jumper should land with the same foot placement after each jump and land softly each time. The effort of the athlete should not decline prior to the completion of the 10 seconds. The instructions to raters differ between studies. However, all 5 raters in the study by Dudley et $\mathrm{al}^{9}$ received the same instructions, videos, and original Myer et $\mathrm{al}^{7}$ article that describes the scoring. The raters in the study by Dudley et $\mathrm{al}^{9}$ were allowed to watch the videos as many times as necessary. The raters scored each video independently, with no discussion allowed.

Scoring of the TJA is quick but not necessarily simple, with 10 flaws to score. The 10 flaws include LE valgus at landing, thighs not reaching parallel, thighs not equal side to side, foot placement not shoulder width apart, foot placement not parallel (front to back), foot contact timing not equal, excessive landing contact noise, pause between jumps, technique declines prior to 10 seconds, and does not land in the same foot placement. ${ }^{5-7}$ The different flaws must be viewed from either the frontal or sagittal plane and with the volume on. It can be difficult to discern whether a flaw is present or not, especially when rating videos at full speed. Because of the difficulty of scoring a flaw, scorers are likely to slow down the video replay speed in order to view the flaws unless given specific instructions not to. To maintain consistency between scoring attempts, there is a need for standardization of video playback speed because some flaws may be better viewed - and, therefore, better scored - at different speeds. There is also a need for standardization for the video volume because one of the flaws is excessive contact noise. Currently, there is no recommendation on the video playback speed or volume used when viewing the videos.

Two different scoring systems exist for the TJA, the original version and the modified version. ${ }^{5-7,12}$ The original scoring system 


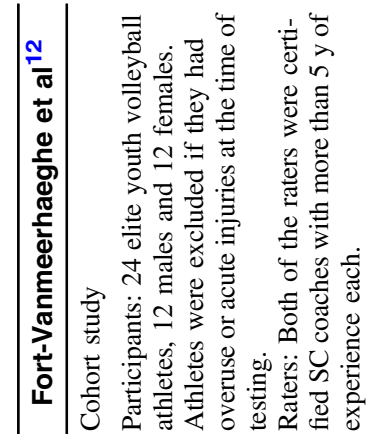

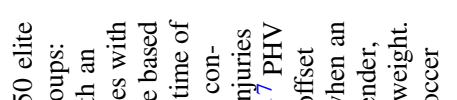

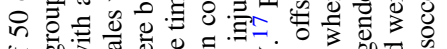

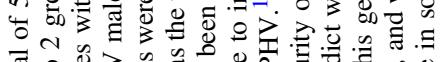

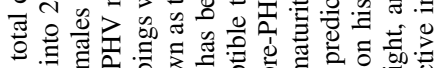

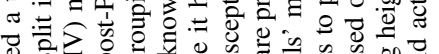

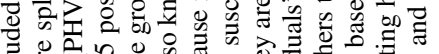

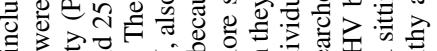

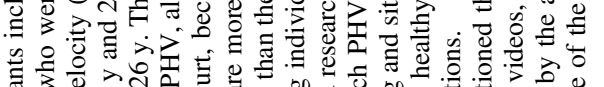

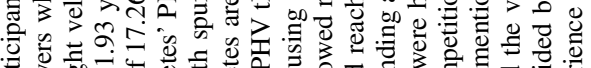

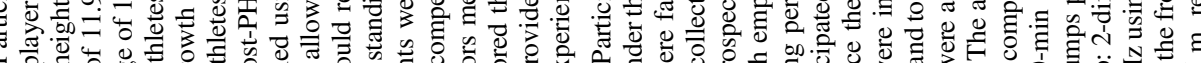

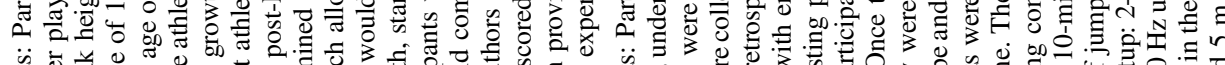

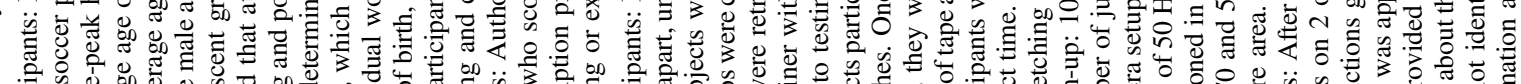

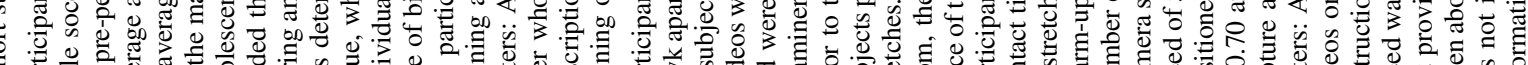

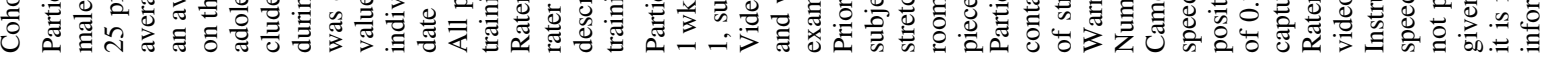

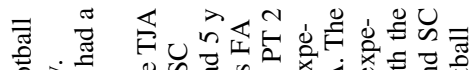

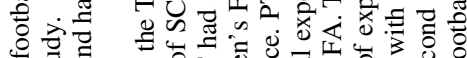

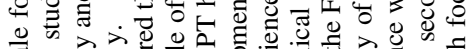

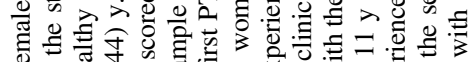

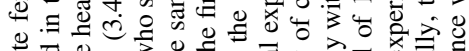
氜啳

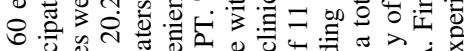

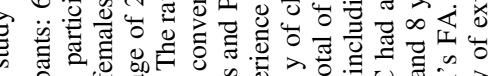

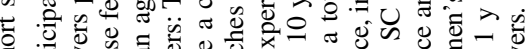

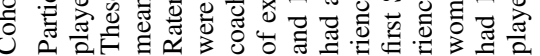

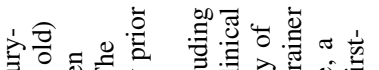

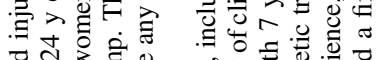

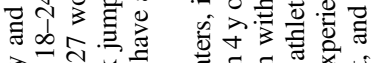

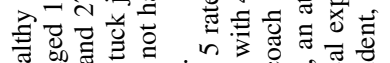

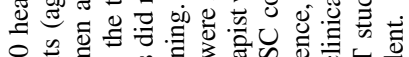

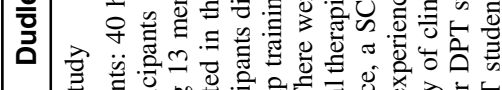

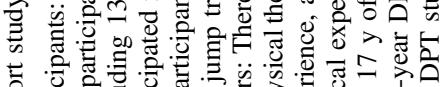

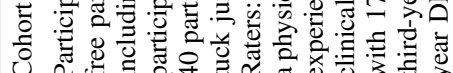
จ
论
入苟诺
可 글 巳

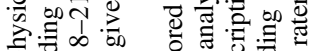

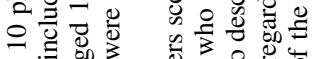

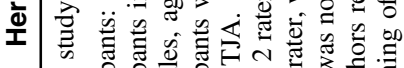

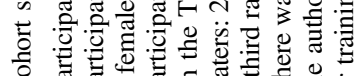

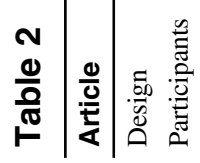

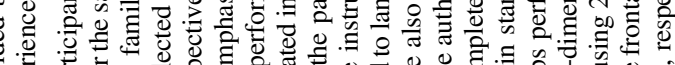

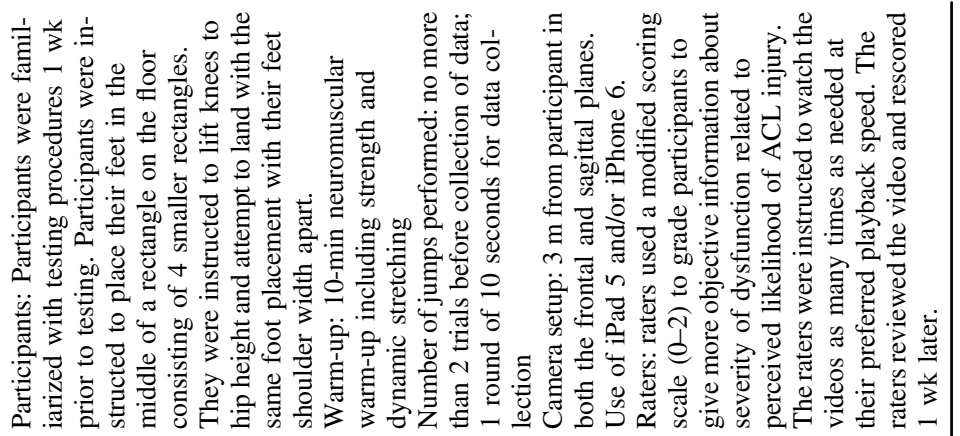

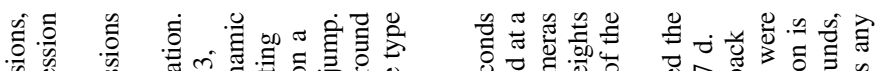
焉

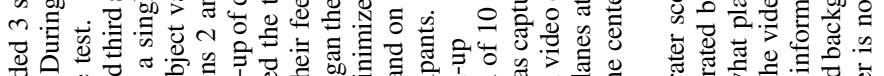

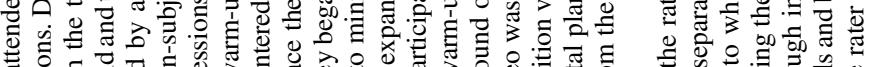

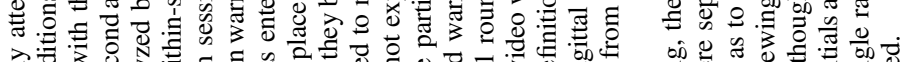

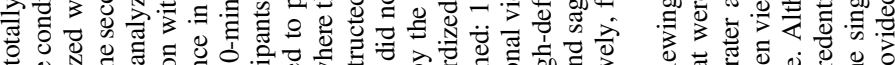

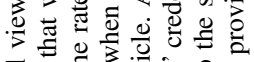
西记

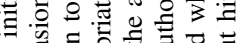

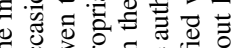

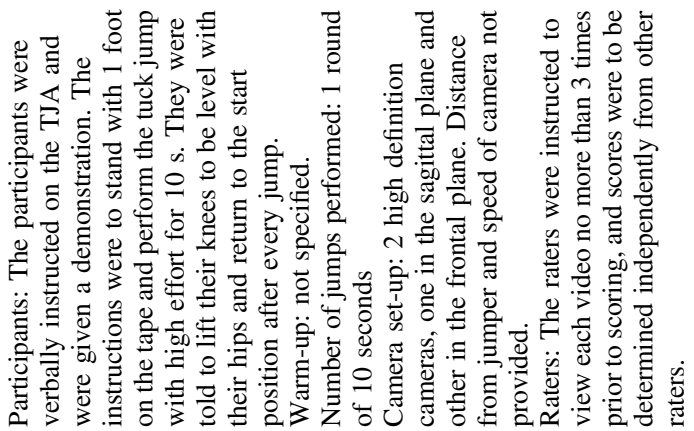

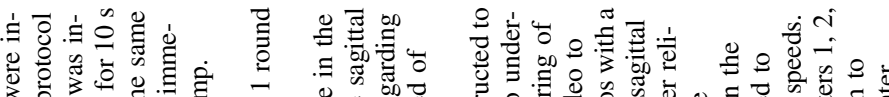

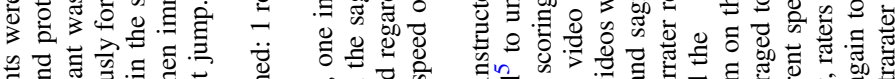

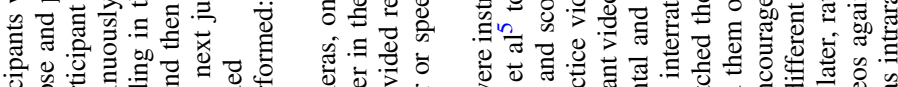

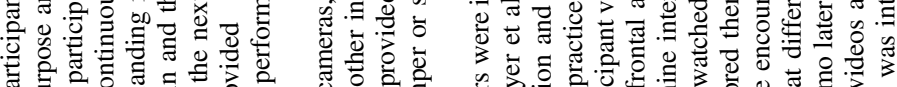

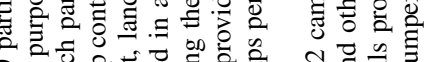

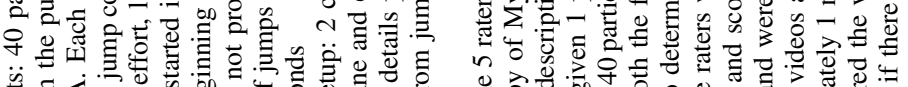

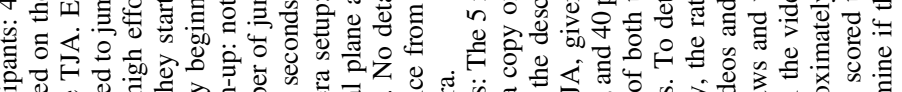

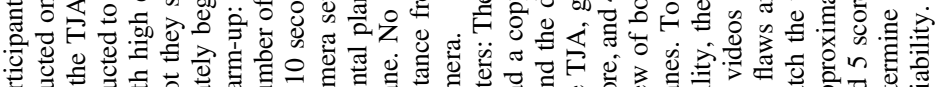

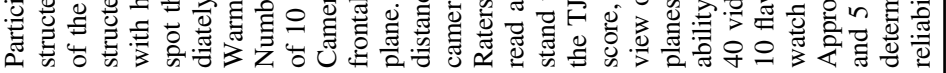

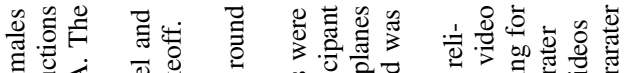

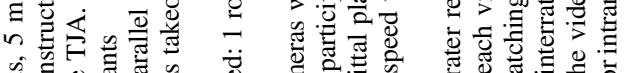

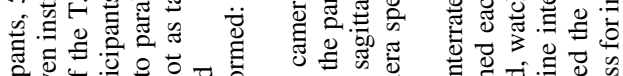
:

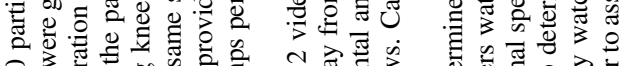

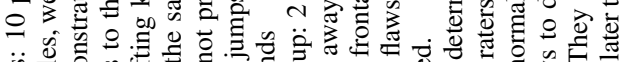

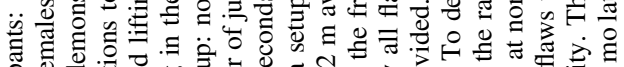

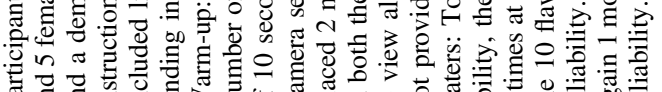

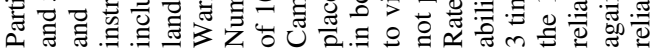




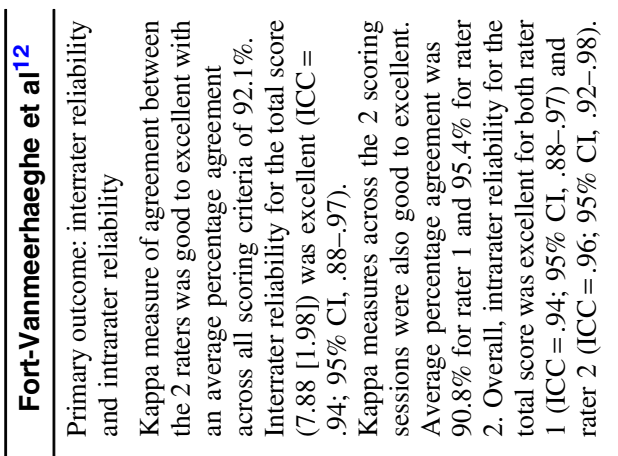

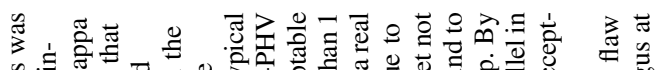

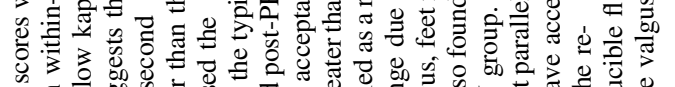

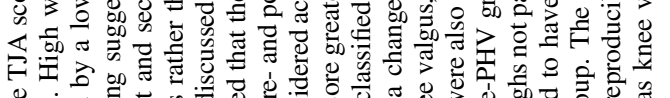

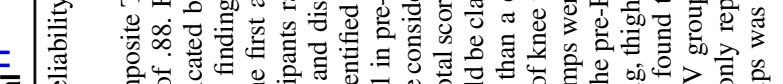

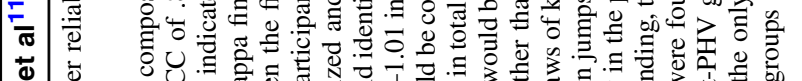

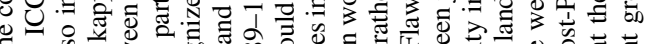

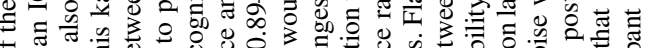

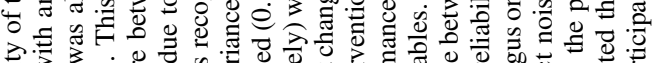

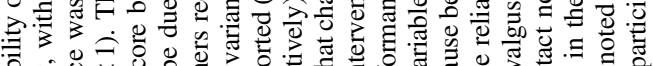

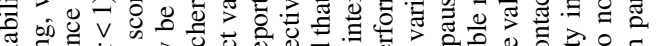

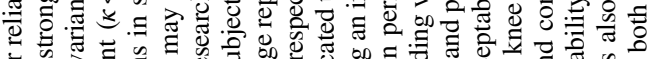

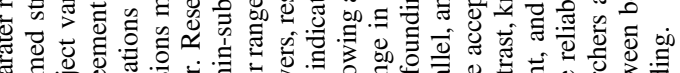

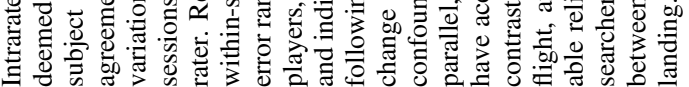

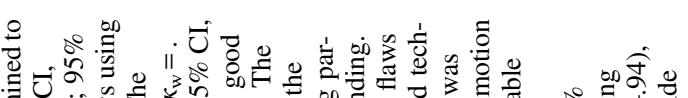

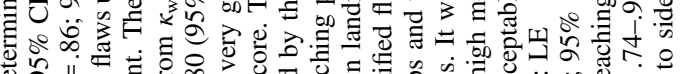

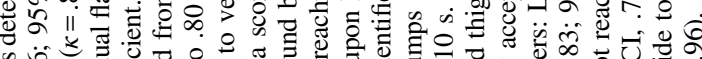

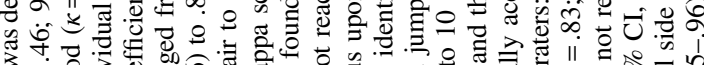

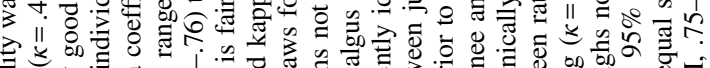

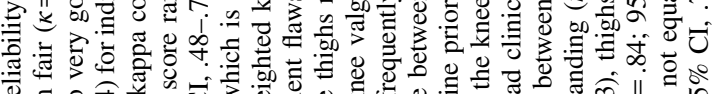

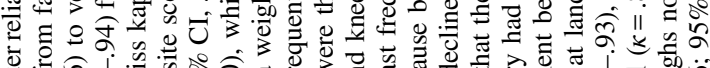

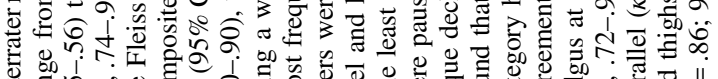

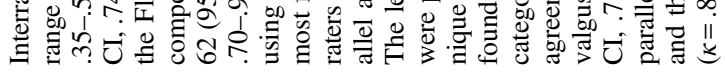

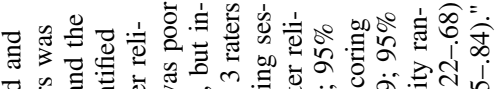

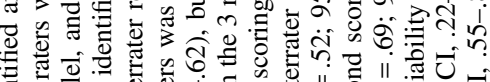

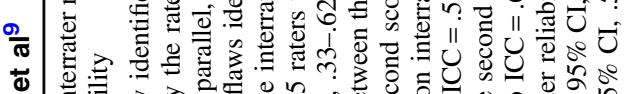

屯

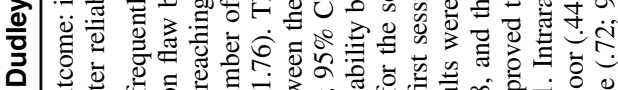

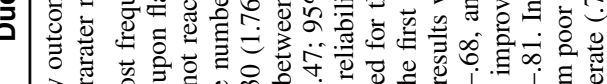

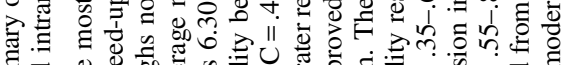

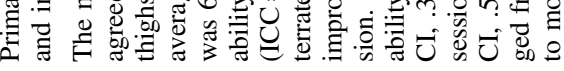

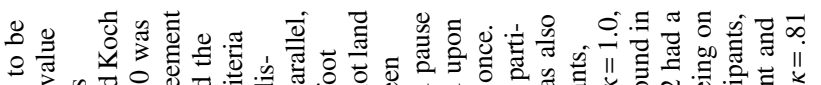

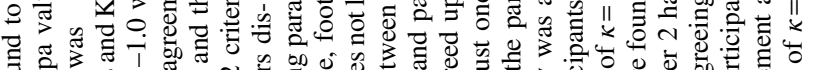

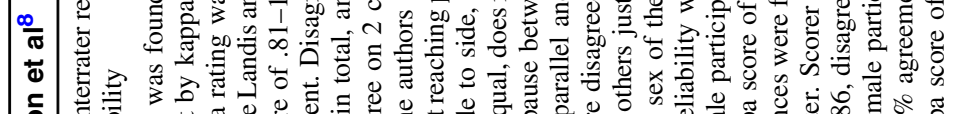
б.

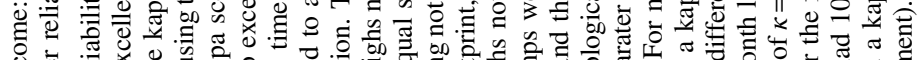

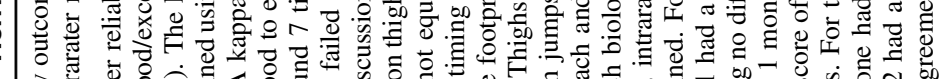

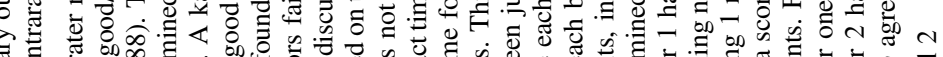

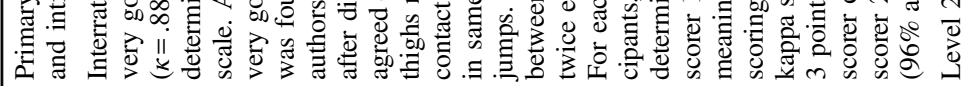

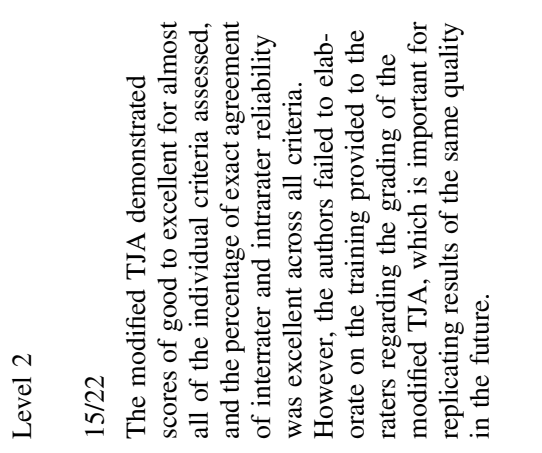

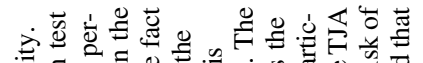

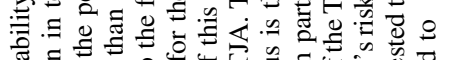

焉.

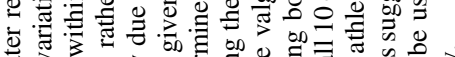

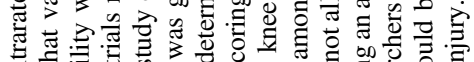

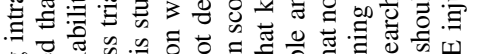

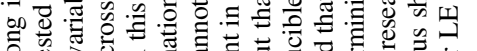

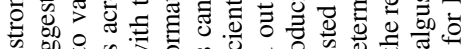

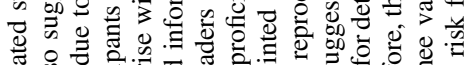

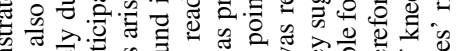

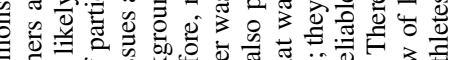

훈

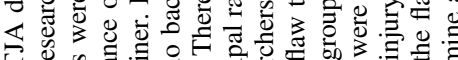

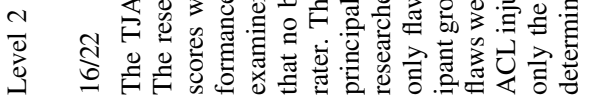

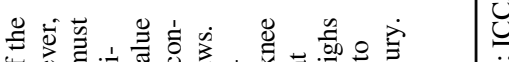

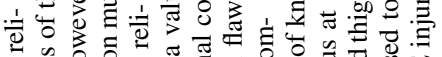

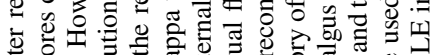

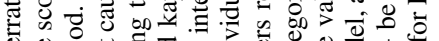

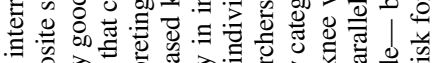

응

至

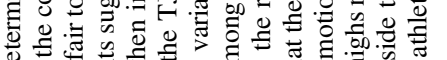

क्ष

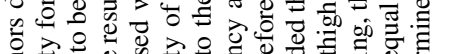

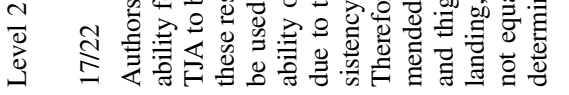

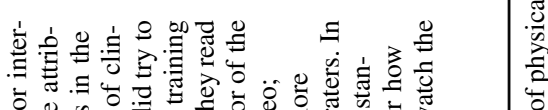

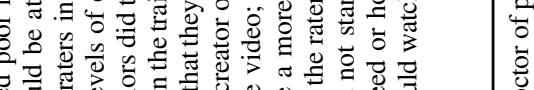

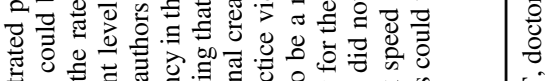

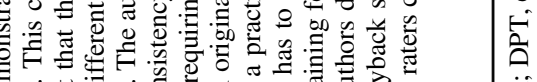

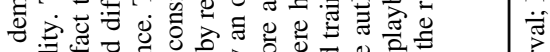

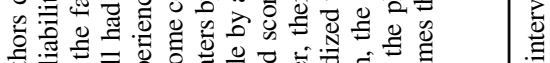

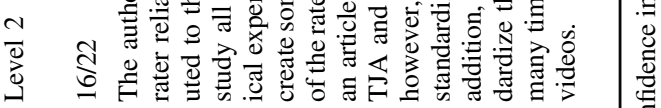

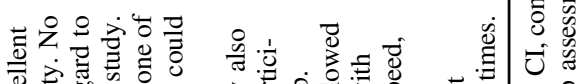

ช

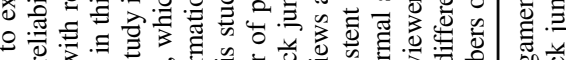

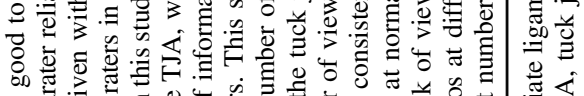

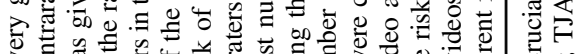

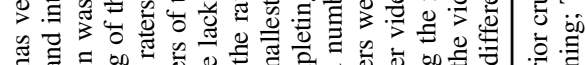

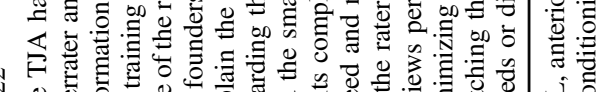

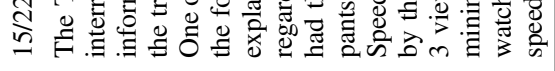


consists of 2 options; criteria are either met or not met. ${ }^{5-7}$ By contrast, the modified scoring system allows the rater to determine how severe the flaw is, giving 3 options for scoring: 0,1 , or $2 .{ }^{12}$ Reliability in scoring the TJA can be altered based on the use of different scoring systems. The option of determining the severity of a flaw can allow for more specialized training to correct the flaw based on modifiable risk factors identified by Myer et $\mathrm{al}^{7}$ and Lininger et $\mathrm{al}^{16}$; however, this scoring system also introduces a greater degree of subjectivity when scoring the TJA. Standardization of scoring is needed in order to provide raters with the preferred scoring system.

In the research used for this study, reliability was found to be good to excellent in $4^{8,10-12}$ of the 5 studies. However, 2 of the studies that deemed that the TJA had either strong intrarater reliability (intraclass correlation $=.88)^{11}$ or fair to good interrater reliability $\left(\kappa_{\mathrm{w}}=.62-.80\right),{ }^{10}$ and stated that although the composite score of the TJA demonstrated reliability, caution should be taken when interpreting the total score as there is high within-subject variation across a number of flaws. ${ }^{11}$ Statistical analysis to determine if scores for each of the 10 flaws were reproducible independent of one another concluded that only 1 flaw demonstrated substantial agreement in both prepeak height velocity and postpeak height velocity youth athletes $(\kappa=.78$ and .67, respectively), that is, knee valgus at landing. ${ }^{11}$ Thus, the authors suggested that not all 10 flaws scored on the TJA were reliable enough to determine an athlete's risk for ACL or LE injury and that perhaps only the flaw of knee valgus at landing should be used. $^{11}$ The Mayhew et al ${ }^{10}$ study also concluded that the interrater reliability of individual flaws varied from fair to very good $(\kappa=.46-.86)$ and demonstrated that knee valgus at landing, thighs not parallel, and thighs not equal side to side were found to have the best reliability among the flaws. Standardization of particular variables must be done for future research in order to determine the true reliability of the TJA. Another consideration with regard to the published literature on the reliability of the TJA is that the original author of the TJA was an author and sometimes a rater for 3 of the 5 articles that demonstrated good to excellent reliability ${ }^{8,11,12}$; this may serve as a source of potential bias. In addition, one of the studies that indicated that the TJA demonstrated good to excellent reliability had the lowest number of participants in the study and, therefore, the lowest power of any of the studies examined. ${ }^{10}$ Fort-Vanmeerhaeghe et al ${ }^{12}$ demonstrated strong reliability for the TJA; however, they used a modified scoring system. This presents another inconsistency in the available research as the modified scoring system rates TJA flaws differently than the other studies reviewed. Although Dudley et $\mathrm{al}^{9}$ determined poor reliability overall, the reliability increased on the second viewing of the videos, suggesting a possible learning curve. All of the studies that were assessed and presented data to support the reliability of the TJA also contained confounding variables of results and potential sources of bias. ${ }^{8,10-12}$ Therefore, based on the scarce body of evidence currently available, a clear decision cannot be determined as to whether the TJA is reliable in healthy subjects in terms of interrater and intrarater reliability.

\section{Acknowledgment}

The authors have no conflicts of interest to disclose.

\section{References}

1. Malone TR, Hardaker WT, Garrett WE, Feagin JA, Bassett FH. Relationship of gender to anterior cruciate ligament injuries in intercollegiate basketball players. $J$ South Orthop Assoc. 1993;2(1):36-39.

2. Griffin LY, Agel J, Albohm MJ, et al. Noncontact anterior cruciate ligament injuries: risk factors and prevention strategies. $J$ Am Acad Orthop Sur. 2000;8(3):141-150. doi:10.5435/00124635-20000500000001

3. Padua DA, Marshall SW, Boling MC, Thigpen CA, Garrett WE Jr, Beutler AI. The Landing Error Scoring System (LESS) is a valid and reliable clinical assessment tool of jump-landing biomechanics: the JUMP-ACL study. Am J Sports Med. 2009;37(10):1996-2002. PubMed ID: 19726623 doi:10.1177/0363546509343200

4. Barber-Westin SD, Smith ST, Campbell T, Noyes FR. The drop-jump video screening test: retention of improvement in neuromuscular control in female volleyball players. Strength Cond J. 2010; 24(11):3055-3062. doi:10.1519/JSC.0b013e3181d83516

5. Myer GD, Paterno MV, Ford KR, Quatman CE, Hewett TE. Rehabilitation after anterior cruciate ligament reconstruction: criteria-based progression through the return-to-sport phase. J Orthop Sports Phys Ther. 2006;36(6):385-402. PubMed ID: 16776488 doi:10.2519/jospt. 2006.2222

6. Myer GD, Ford KR, Hewett TE. Tuck jump assessment for reducing anterior cruciate ligament injury risk. Athl Ther Today. 2008;13(5):39-44. PubMed ID: 19936042 doi:10.1123/ att.13.5.39

7. Myer GD, Brent JL, Ford KR, Hewett TE. Real-time assessment and neuromuscular training feedback techniques to prevent ACL injury in female athletes. Strength Cond J. 2012;33(3):21-35. doi:10.1519/ SSC.0b013e318213afa8

8. Herrington L, Myer GD, Munro A. Intra and inter-tester reliability of the tuck jump assessment. Phys Ther Sport. 2013;14:152-155. PubMed ID: 23084318 doi:10.1016/j.ptsp.2012.05.005

9. Dudley LA, Smith CA, Olson BK, Chimera NJ, Schmitz B, Warren M. Interrater and intrarater reliability of tuck jump assessment by health professionals of varied educational backgrounds. J Sports Med. 2013;2013:1-5. doi:10.1155/2013/483503

10. Mayhew L, Johnson MI, Francis P, Snowdon N, Jones G. Inter-rater reliability, internal consistency and common technique flaws of the tuck jump assessment in elite female football players. Sci Med Football. 2017;1(2):139-144. doi:10.1080/24733938.2017. 1282165

11. Read P, Oliver JL, De Ste Croix MBA, Myer GD, Lloyd RS. Reliability of the tuck jump injury risk screening assessment in elite male youth soccer players. J Strength Cond Res. 2016;30(6):1510 1516. doi:10.1519/JSC.0000000000001260

12. Fort-Vanmeerhaeghe A, Montalvo AM, Lloyd RS, Read P, Myer GD. Intra- and inter-rater reliability of the modified tuck jump assessment. J Sports Sci Med. 2017;16(1):117-124. PubMed ID: 28344460

13. Peterson-Kaiser Health System Tracker. How much does the U.S. spend to treat different diseases? https://www.healthsystemtracker. org/chart-collection/much-u-s-spend-treat-different-diseases/\#itemstart. Accessed November 15, 2018.

14. Padua D, DiStefano L, Hewett T, et al. National Athletic Trainers' Association position statement: prevention of anterior cruciate ligament injury. J Athl Train. 2018;53(1):5-19. PubMed ID: 29314903 doi:10.4085/1062-6050-99-16 
15. Herzog MM, Marshall SW, Lund JL, Pate V, Spang JT. Cost of outpatient arthroscopic anterior cruciate ligament reconstruction among commercially insured patients in the United States, 20052013. Orthop J Sports Med. 2017;5(1):2325967116684776. PubMed ID: 28210655 doi:10.1177/2325967116684776

16. Lininger MR, Smith CA, Chimera NJ, Hoog P, Warren M. Tuck jump assessment: an exploratory factor analysis in a college age population. Strength Cond J. 2017;31(3):653-659. doi:10.1519/JSC. 0000000000001186

17. Van der Sluis A, Elferink-Gemser MT, Coelho-e-Silva MJ, Nijboer JA, Brink MS, Visscher C. Sport injuries aligned to peak height velocity in talented pubertal soccer players. Int J Sports Med. 2014;35(4):351-355. doi:10.1055/s-00331349874 